P. Valabrega and G. Valla

Nagoya Math. J.

Vol. 72 (1978), 93-101

\title{
FORM RINGS AND REGULAR SEQUENCES ${ }^{(1)}$
}

\author{
PAOLO VALABREGA AND GIUSEPPE VALLA
}

\section{Introduction}

Hironaka, in his paper $\left[\mathrm{H}_{1}\right]$ on desingularization of algebraic varieties over a field of characteristic 0 , to deal with singular points develops the algebraic apparatus of the associated graded ring, introducing standard bases of ideals, numerical characters $\nu^{*}$ and $\tau^{*}$ etc. Such a point of view involves a deep investigation of the ideal $b^{*}$ generated by the initial forms of the elements of an ideal $\boldsymbol{b}$ of a local ring, with respect to a certain ideal $a$.

The present paper has its origin in the effort of extending to a general situation the following result (due to Hironaka: $\left[\mathrm{H}_{2}\right]$ ):

Let $(A, \boldsymbol{m})$ be a local ring and $z \in \boldsymbol{m}-\boldsymbol{m}^{2}$; then the initial form $z^{*}$ of $z$ in the associated graded ring $G(\boldsymbol{m})$ is a regular element if and only if $z$ is regular in $A$ and $(z) \cap \boldsymbol{m}^{n+1}=(z) \cdot \boldsymbol{m}^{n}$, for every integer $n$.

Really our paper investigates in a general way the relations between an ideal $\boldsymbol{b}=\left(f_{1}, \cdots, f_{r}\right)$ and the associated graded ideal $\boldsymbol{b}^{*}$ generated by the initial forms of the elements of $\boldsymbol{b}$ in $G_{A}(\boldsymbol{a})=$ graded ring with respect to the ideal $a$ of the ring $A$.

We prove the following main results:

1-a necessary and sufficient condition for $b^{*}$ to be generated by the initial forms of the $f_{i}$ 's, valid for an arbitrary noetherian ring $A$;

2-a necessary and sufficient condition for $\boldsymbol{b}^{*}$ to be generated by a regular sequence, valid for an arbitrary noetherian ring $A$;

3-a condition like in 2, valid for a local ring $A$, and generalizing in a natural way Hironaka's result.

The paper contains also some other properties of the associated graded ideal (concerning height and minimal generating sets) and of $G_{A}(\boldsymbol{a})$ (conditions to be Cohen-Macaulay: see also [S]).

Received September 16, 1977.

(1) The present paper has been written while both authors were members of CNR, section 3 of GNSAGA. 


\section{Form rings and ideals}

If $A$ is a noetherian ring with a unit element 1 and $a$ any ideal in $A$, we denote by $G_{A}(\boldsymbol{a})$ the graded $A / \boldsymbol{a}$-algebra $\bigoplus_{n=0}^{\infty} \boldsymbol{a}^{n} / \boldsymbol{a}^{n+1}$ and call it the form ring of $A$ relative to $a$ (other term: associated graded ring).

Sometimes we will have to deal also with negative powers of the ideal $a$; once for all $\boldsymbol{a}^{n}=A$ if $n \leq 0$.

Given an element $a \in A$, we denote by $v(a)$ the largest integer $n$ such that $a \in \boldsymbol{a}^{n}$; if $a \in \bigcap_{n=1}^{\infty} \boldsymbol{a}^{n}$ we set: $v(a)=\infty$. When $v(a) \neq \infty$ the residue class of $a$ in $\boldsymbol{a}^{v(a)} / \boldsymbol{a}^{v(a)+1}$ is called the initial form of $a$ and denoted by $a^{*}$. If $v(a)=\infty$, then we set: $a^{*}=0$.

The definition of multiplication in $G_{A}(a)$ shows that the two relations: $a^{*} b^{*}=(a b)^{*}$ and $a^{*} b^{*} \neq 0$ are equivalent, provided that $\boldsymbol{a}^{*}$ and $\boldsymbol{b}^{*} \neq 0$.

Let now $\boldsymbol{b}$ be any ideal of $A$; we shall denote by $\boldsymbol{b}^{*}$ the homogeneous ideal of $G_{A}(a)$ generated by all the initial forms of the elements in $\boldsymbol{b} ; \boldsymbol{b}^{*}$ is called the form ideal of $\boldsymbol{b}$ relative to $\boldsymbol{a}$ (or the associated graded ideal). For every integer $n$ we have: $\boldsymbol{b}_{n}^{*}=$ set of homogeneous elements in $\boldsymbol{b}^{*}$ of degree $n=\left(\boldsymbol{b} \cap \boldsymbol{a}^{n}+\boldsymbol{a}^{n+1}\right) / \boldsymbol{a}^{n+1}$; furthermore $\boldsymbol{b}^{*}$ is the kernel of a natural epimorphism of graded rings

$$
\varphi: G_{A}(\boldsymbol{a}) \rightarrow G_{A / \boldsymbol{b}}(\boldsymbol{b}+\boldsymbol{a} / \boldsymbol{b}),
$$

which is homogeneous of degree 0 .

The ideal $\boldsymbol{b}^{*}$ can be defined with respect to any other ideal $\boldsymbol{a}$; but, if $\boldsymbol{b}$ and $\boldsymbol{a}$ are comaximal (i.e. $\boldsymbol{a}+\boldsymbol{b}=A$ ) then $\boldsymbol{b}^{*}=G_{A}(\boldsymbol{a})$ and conversely (as one may easily check). Therefore we assume, once for all, that a and $\boldsymbol{b}$ are not comaximal. Of course both $\boldsymbol{a}$ and $\boldsymbol{b}$ are assumed to be proper.

Since $A$ is noetherian, then $G_{A}(a)$ is noetherian. In particular $b^{*}$ is generated by the initial forms of a finite number of elements of $\boldsymbol{b}$. However it is not generally true that, if $\boldsymbol{b}=\left(f_{1}, \cdots, f_{r}\right)$, then $\boldsymbol{b}^{*}$ $=\left(f_{1}^{*}, \cdots, f_{r}^{*}\right)$.

The following theorem is just a necessary and sufficient condition for the equality:

THEOREM 1.1. If $\boldsymbol{a}$ and $\boldsymbol{b}=\left(f_{1}, \cdots, f_{r}\right)$ are ideals of $A$, then $\boldsymbol{b}^{*}$ $=\left(f_{1}^{*}, \ldots, f_{r}^{*}\right)$ in $G_{A}(a)$ if and only if for all $n \geqslant 1$ the following equality holds : 


$$
\boldsymbol{a}^{n} \cap \boldsymbol{b}=\sum_{i=1}^{r} \boldsymbol{a}^{n-p_{i}} f_{i}
$$

where $p_{i}=v\left(f_{i}\right), i=1, \cdots, r$.

Proof. It is clear that $\left(f_{1}^{*}, \cdots, f_{r}^{*}\right)_{n}=\left(\sum_{i=1}^{r} \boldsymbol{a}^{n-p_{i}} f_{i}+\boldsymbol{a}^{n+1}\right) / \boldsymbol{a}^{n+1}$.

Hence, if $\boldsymbol{b} \cap \boldsymbol{a}^{n}=\sum_{i=1}^{r} \boldsymbol{a}^{n-p_{i}} f_{i}$ for all $n \geqslant 1$, we have: $\boldsymbol{b}^{*}=$ $\left(f_{1}^{*}, \cdots, f_{r}^{*}\right)$.

Conversely, if $\boldsymbol{b}^{*}$ is generated by the $f_{i}^{* \prime}$, then we have: $\boldsymbol{a}^{n} \cap \boldsymbol{b}$ $\subseteq \sum_{i=1}^{r} \boldsymbol{a}^{n-p_{i}} f_{i}+\boldsymbol{a}^{n+1}$ for all $n \geqslant 1$; it follows that $\boldsymbol{a}^{n} \cap \boldsymbol{b}=$ $\bigcap_{t=1}^{\infty}\left(\sum_{i=1}^{r} \boldsymbol{a}^{n-p_{i}} f_{i}+\boldsymbol{a}^{n+t} \cap \boldsymbol{b}\right)$. By the Artin-Rees lemma there exists an integer $q \geqslant 0$ such that $\boldsymbol{a}^{n+t} \cap \boldsymbol{b}=\boldsymbol{a}^{n+t-q}\left(\boldsymbol{a}^{q} \cap \boldsymbol{b}\right)$ for all $n+t \geqslant q$. Hence, if $d$ is an integer such that $d \geqslant n-p_{i}$ for $i=1, \cdots, r$, we get the following equality:

$$
\boldsymbol{a}^{n} \cap \boldsymbol{b}=\bigcap_{t \geqslant q-n+d}\left(\sum_{i=1}^{r} \boldsymbol{a}^{n-p_{i}} f_{i}+\boldsymbol{a}^{n+t-q}\left(\boldsymbol{a}^{q} \cap \boldsymbol{b}\right)\right)=\sum_{i=1}^{r} \boldsymbol{a}^{n-p_{i}} f_{i},
$$

since, if $t \geqslant q-n+d$, then $\boldsymbol{a}^{n+t-q}\left(\boldsymbol{a}^{q} \cap \boldsymbol{b}\right) \subseteq \boldsymbol{a}^{d} \boldsymbol{b} \subseteq \sum_{i=1}^{r} \boldsymbol{a}^{n-p_{i}} f_{i}$.

Remark 1.2. Using [R, Rem. (3.7)] one can easily see (cf. [V]) that, if $\boldsymbol{a}$ and $\boldsymbol{b}$ are ideals of $\boldsymbol{A}$ such that $\boldsymbol{a}+\boldsymbol{b} \neq \boldsymbol{A}$, then $h\left(\boldsymbol{b}^{*}\right) \geqslant h(\boldsymbol{b})$ at least when $A$ is local, and certainly equality holds whenever $\boldsymbol{a} \subseteq \boldsymbol{b}$; moreover there are examples with strict inequality. Therefore the following result may have some interest:

Proposition 1.3. If $\boldsymbol{a}$ and $\boldsymbol{b}=\left(f_{1}, \cdots, f_{r}\right)$ are ideals of $A$ such that $\boldsymbol{a}+\boldsymbol{b} \neq A$, then $h\left(\boldsymbol{b}^{*}\right) \leqslant r$.

Proof. Let $\boldsymbol{m}$ be a maximal ideal containing both $\boldsymbol{a}$ and $\boldsymbol{b}$; then we have : $h(\boldsymbol{m})-\boldsymbol{r} \leq h(\boldsymbol{m} / \boldsymbol{b})=h\left((\boldsymbol{m} / \boldsymbol{b})^{*}\right)=h\left(\boldsymbol{m}^{*} / \boldsymbol{b}^{*}\right) \leq h\left(\boldsymbol{m}^{*}\right)-h\left(\boldsymbol{b}^{*}\right)=h(\boldsymbol{m})$ $-h\left(b^{*}\right)$ (in the preceding chain of inequalities we use the fact that, in the isomorphism $G_{A}(a) / b^{*} \cong G_{A / b}(a+b / b), m^{*} / b^{*}$ and $(m / b)^{*}$ are corresponding ideals).

Remark 1.4. If $h\left(f_{1}^{*}, \cdots, f_{r}^{*}\right)=r$, then also $h\left(\boldsymbol{b}^{*}\right)=r$. However the following example shows that the condition $h\left(f_{1}^{*}, \cdots, f_{r}^{*}\right)=r$ does not imply the equality $\boldsymbol{b}^{*}=\left(f_{1}^{*}, \cdots, f_{r}^{*}\right)$. 
EXAMPLE 1.5. Let $A$ be the ring $k\left[\left[t^{4}, t^{5}, t^{11}\right]\right]=k[[X, Y, Z]] /(X Z-$ $\left.Y^{3}, Y Z-X^{4}, Z^{2}-X^{3} Y^{2}\right)=k[[x, y, z]], \quad \boldsymbol{a}=(x, y, z), \quad \boldsymbol{b}=(x)$; we have: $G_{A}(\boldsymbol{a})=k\left[T_{1}, T_{2}, T_{3}\right] /\left(T_{1} T_{3}, T_{2} T_{3}, T_{3}^{2}, T_{2}^{4}\right)=k\left[t_{1}, t_{2}, t_{3}\right] ;$ hence $h\left(\boldsymbol{b}^{*}\right)=h\left(t_{1}\right)=$ 1, but $y^{3} \in \boldsymbol{a}^{3} \cap \boldsymbol{b}$ and $y^{3} \notin \boldsymbol{a}^{2} x$ and so, by Theorem 1.1, $\boldsymbol{b}^{*} \neq\left(x^{*}\right)$.

\section{Regular sequences in $G_{A}(a)$}

In this section we consider a noetherian ring $A$ and an ideal $\boldsymbol{a}$ in $A$; if $f_{1}, \cdots, f_{r} \in A$ we shall state some necessary and sufficient conditions for $f_{1}^{*}, \cdots, f_{r}^{*}$ to be a regular sequence in $G_{A}(a)$ (always provided that $\boldsymbol{a}$ and $\left(f_{1}, \cdots, f_{r}\right)$ be not comaximal).

In the following we shall write $p_{i}=v\left(f_{i}\right)$ and $\boldsymbol{b}_{i}=\left(f_{1}, \cdots, f_{i}\right)$, for $i=1, \cdots, r\left(b_{0}=(0)\right)$.

Proposition 2.1. If $\boldsymbol{a}$ and $\boldsymbol{b}=\left(f_{1}, \cdots, f_{r}\right)$ are ideals of $A$ such that $f_{1}^{*}, \cdots, f_{r}^{*}$ is a $G_{A}(a)$-sequence, then $\boldsymbol{b}^{*}=\left(f_{1}^{*}, \cdots, f_{r}^{*}\right)$.

Proof. We use induction on $r$. The case $r=1$ is easy: if $f^{*}$ is a non zero-divisor, then, for every $g,(f g)^{*}=f^{*} g^{*}$, which shows our claim. Assume now the theorem true for $r-1$ and prove it for $r$.

If $a \in \boldsymbol{a}^{n} \cap \boldsymbol{b}$ let $t$ be the greatest integer (if it exists, $\infty$ if it does not exist) such that $a \in \boldsymbol{b}_{r-1}+f_{r} \boldsymbol{a}^{t}$. So we can write $a=x+f_{r} y$, with $v(y)=t, x \in \boldsymbol{b}_{r-1}$; if $t+p_{r}<n$ we have: $f_{r} y \in\left(\boldsymbol{a}^{n}+\boldsymbol{b}_{r-1}\right) \cap \boldsymbol{a}^{t+p_{r}} \subseteq \boldsymbol{a}^{t+p_{r+1}}$ $+\boldsymbol{b}_{r-1} \cap \boldsymbol{a}^{t+p_{r}}$.

It follows that $f_{r}^{*} y^{*} \in \boldsymbol{b}_{r-1}^{*}$; by our inductive hypothesis $\boldsymbol{b}_{r-1}^{*}$ is generated by the initial forms of the $f_{i}$ 's, $i=1, \cdots, r-1$, hence $y^{*} \in \boldsymbol{b}_{r-1}^{*}$. Then $y \in \boldsymbol{a}^{t+1}+\boldsymbol{b}_{r-1} \cap \boldsymbol{a}^{t}$, so that $a \in \boldsymbol{b}_{r-1}+f_{r} \boldsymbol{a}^{t+1}$, which is absurd. Therefore $t+p_{r} \geqslant n$, hence $a \in \boldsymbol{a}^{n} \cap \boldsymbol{b}_{r-1}+f_{r} \boldsymbol{a}^{n-p_{r}}$. The conclusion follows immediately from the inductive assumption together with our Theorem 1.1.

Remark 2.2. The converse of Proposition 2.1 is false even if $A$ is local. In fact, let $A=k[[X, Y]] /(X Y)=k[[x, y]], \quad \boldsymbol{b}=(x), \quad \boldsymbol{a}=(x, y)$; then we have: $\boldsymbol{a}^{n} \cap \boldsymbol{b}=\left(x^{n}, y^{n}\right) \cap(x)=\left(x^{n}\right)+\left(y^{n}\right) \cap(x)=\left(x^{n}\right)=\boldsymbol{a}^{n-1} \boldsymbol{b}$. Hence by Theorem $1.1 b^{*}=\left(x^{*}\right)$, but $G_{A}(a)=k\left[T_{1}, T_{2}\right] /\left(T_{1} T_{2}\right)=k\left[t_{1}, t_{2}\right]$ and $x^{*}=t_{1}$ is a zero divisor in $G_{A}(\boldsymbol{a})$.

In the following we shall denote by $\bar{I}$ the topological closure of an ideal $I$ with respect to the $\boldsymbol{a}$-adic topology. 
THEOREM 2.3. Let $\boldsymbol{a}$ and $\boldsymbol{b}=\left(f_{1}, \cdots, f_{r}\right)$ be ideals of the noetherian ring $A$. Then the following facts are equivalent:

(i) $\left(f_{1}^{*}, \cdots, f_{r}^{*}\right)$ is a $G_{A}($ a $)$-sequence;

(ii) for each $i=1, \cdots, r, b_{i-1}: f_{i} \subseteq \overline{\boldsymbol{b}}_{i-1}$ and $\boldsymbol{b}_{i} \cap \boldsymbol{a}^{n}=\sum_{j=1}^{i} \boldsymbol{a}^{n-p_{j}} f_{j}$, for all $n \geqslant 1$.

Proof. (i) $\Rightarrow$ (ii). For Proposition 2.1 it is enough to show that $\boldsymbol{b}_{i-1}: f_{i} \subseteq \overline{\boldsymbol{b}}_{i-1}$. Let $a \in \boldsymbol{b}_{i-1}: f_{i}$ with $v(a)=n$; then $a^{*} f_{i}^{*} \in \boldsymbol{b}_{i-1}^{*}=\left(f_{1}^{*}\right.$, $\left.\cdots, f_{i-1}^{*}\right)$, hence $a^{*} \in \boldsymbol{b}_{i-1}^{*}$. It follows that $a \in \boldsymbol{b}_{i-1}+\boldsymbol{a}^{n+1} \cap\left(\boldsymbol{b}_{i-1}: f_{i}\right)$; repeating the argument we see that $a \in \bar{b}_{i-1}$.

(ii) $\Rightarrow$ (i). Conversely, since $\boldsymbol{a}+\boldsymbol{b} \neq A$ and $\boldsymbol{b}_{i-1}: f_{i} \subseteq \overline{\boldsymbol{b}}_{i-1}$ for each $i=1, \cdots, r$, we have $p_{i}<\infty$ for each $i=1, \cdots, r$. In fact, assume that $p_{i}=\infty$ for some $i$; then there exists $a \in a$ such that $(1-a) f_{i}=0$. Thus $1-a \in \bar{b}_{i-1}$ and then $(1-a)\left(1-a^{\prime}\right) \in \boldsymbol{b}_{i-1}$, with $a^{\prime} \in \boldsymbol{a}$; but since $\boldsymbol{a}+\boldsymbol{b} \neq A$, this is a contradiction.

Now let $a^{*} f_{i}^{*} \in\left(f_{1}^{*}, \cdots, f_{i-1}^{*}\right)$ with $v(a)=n$; then $a f_{i} \in \boldsymbol{b}_{i-1}+\boldsymbol{a}^{n+p_{i}+1}$, hence $b=a f_{i}+\sum_{j=1}^{i-1} a_{j} f_{j} \in \boldsymbol{b}_{i} \cap \boldsymbol{a}^{n+p_{i}+1}$.

We can write $b=\sum_{j=1}^{i} b_{j} f_{j}$ with $b_{j} \in \boldsymbol{a}^{n+p_{i}+1-p_{j}}$, whence we deduce that $\boldsymbol{a}-b_{i} \in \boldsymbol{b}_{i-1}: f_{i}$ and from this it follows that $a \in \boldsymbol{a}^{n+1}+\overline{\boldsymbol{b}}_{i-1}$.

Finally $a \in \boldsymbol{a}^{n+1}+\boldsymbol{b}_{i-1} \cap \boldsymbol{a}^{n}$ and this proves that $\boldsymbol{a}^{*} \in \boldsymbol{b}_{i-1}^{*}=\left(f_{1}^{*}, \cdots\right.$, $\left.f_{i-1}^{*}\right)$.

Corollary 2.4. Let $A$ be a local ring and I, a ideals of $A$, such that $I^{*}$ is generated by a $G_{A}(\boldsymbol{a})$-sequence. Then $I$ is generated by an A-sequence.

Proof. Let $I^{*}$ be generated by $g_{1}, \cdots, g_{r}$, where the $g_{i}$ 's form a regular sequence. Since all the minimal generating sets of $I^{*}$ have the same number of elements, we can write $\boldsymbol{I}^{*}=\left(f_{1}^{*}, \cdots, f_{r}^{*}\right)$ with $f_{i} \in \boldsymbol{I}$. Now $\operatorname{gr}\left(I^{*}\right)=r$, hence using the homology of Koszul complex we get $H_{1}\left(f_{1}^{*}, \cdots, f_{r}^{*} ; G_{A}(a)\right)=0$. From this it follows that $f_{1}^{*}, \cdots, f_{r}^{*}$ is a $G_{A}(a)$-sequence (see [A-B], Prop. 2.8); hence, by Theorem 2.3, $f_{1}, \cdots, f_{r}$ is an $A$-sequence. Furthermore, since $I^{*}=\left(f_{1}^{*}, \cdots, f_{r}^{*}\right)$, we get: $\left(f_{1}, \cdots, f_{r}\right) \subseteq \boldsymbol{I} \subseteq\left(\overline{\left.f_{1}, \cdots, f_{r}\right)}=\left(f_{1}, \cdots, f_{r}\right)\right.$.

Remark 2.5. If $f_{1}^{*}, \cdots, f_{r}^{*}$ form a regular sequence, it is not necessarily true that $f_{1}, \cdots, f_{r}$ form also an $A$-sequence, unless $\boldsymbol{I}=\overline{\boldsymbol{I}}$ for 
every ideal $I$ contained in $b$. In fact let $A=k[x, y, z]=k[X, Y, Z] /$ $(X Z, X-X Y), a=(y), f=y z$; then, since $y$ is not a 0 -divisor in $A$, we have: $G_{A}(a)=(A / a)[T]=k[Z, T]$ which is a domain. Therefore $\bar{f} \in \boldsymbol{a} / \boldsymbol{a}^{2}$ is not a 0 -divisor in $G_{A}(\boldsymbol{a})$, but $x f=0$.

Proposition 2.6. Let $\boldsymbol{a}$ and $\boldsymbol{b}=\left(f_{1}, \cdots, f_{r}\right)$ be two ideals of $A$ such that $f_{1}, \cdots, f_{r}$ is an A-sequence and $\boldsymbol{a}^{n} \cap \boldsymbol{b}=\sum_{j=1}^{r} \boldsymbol{a}^{n-p_{i}} f_{i}$ for all $n \geqslant 1$. Suppose either $\boldsymbol{b} \subseteq \boldsymbol{a}$ or $A$ is local.

Then $\boldsymbol{a}^{n} \cap \boldsymbol{b}_{i}=\sum_{j=1}^{i} \boldsymbol{a}^{n-p_{j}} f_{j}$, for each $i=1, \cdots, r$ and for all $n \geqslant 1$; thus $f_{1}^{*}, \ldots, f_{r}^{*}$ is a $G_{A}(\boldsymbol{a})$-sequence.

Proof. It is enough to show that $\boldsymbol{a}^{n} \cap \boldsymbol{b}_{r-1}=\sum_{j=1}^{r-1} \boldsymbol{a}^{n-p_{j}} f_{j}$, for all $n \geqslant 1$. Let $a=\sum_{i=1}^{r-1} a_{i} f_{i}$ be an element of $\boldsymbol{a}^{n} ;$ then $a=\sum_{i=1}^{r} b_{i} f_{i}$, where $b_{i} \in \boldsymbol{a}^{n-p_{i}}$ and we get: $b_{r} \in \boldsymbol{a}^{n-p_{r}} \cap \boldsymbol{b}_{r-1}$. Thus we have: $\boldsymbol{a}^{n} \cap \boldsymbol{b}_{r-1} \subseteq \sum_{j=1}^{r-1} \boldsymbol{a}^{n-p_{j}} f_{j}+$ $f_{r}\left(\boldsymbol{a}^{n-p_{r}} \cap \boldsymbol{b}_{r-1}\right)$. Let $m$ be an integer such that $m \leq p_{i}$ for each $i=$ $1, \cdots, r-1$. If $n \leq m+p_{r}$, we have that $\boldsymbol{b}_{r-1} \subseteq \boldsymbol{a}^{n-p_{r}}$ and $f_{r} \in \boldsymbol{a}^{n-p_{i}}$ for each $i=1, \cdots, r-1$; hence $\boldsymbol{a}^{n} \cap \boldsymbol{b}_{r-1}$ is contained in $\sum_{j=1}^{r-1} \boldsymbol{a}^{n-p_{j}} f_{j}+f_{r} \boldsymbol{b}_{r-1}$ $\subseteq \sum_{j=1}^{r-1} \boldsymbol{a}^{n-p_{j}} f_{j}$. Therefore we may assume that $n$ is greater than $m+p_{r}$ and also that $\boldsymbol{a}^{t} \cap \boldsymbol{b}_{r-1}=\sum_{j=1}^{r-1} \boldsymbol{a}^{t-p_{j}} f_{j}$, for all $t \leq n-1$. If $\boldsymbol{b} \subseteq \boldsymbol{a}$, then $p_{r}>0$; if $A$ is local and $p_{r}=0$, then we have $\boldsymbol{a}^{n} \cap \boldsymbol{b}_{r-1} \subseteq \sum_{j=1}^{r-1} \boldsymbol{a}^{n-p_{j}} f_{j}+$ $f_{r}\left(\boldsymbol{a}^{n} \cap \boldsymbol{b}_{r-1}\right)$, hence $\boldsymbol{a}^{n} \cap \boldsymbol{b}_{r-1}=\sum_{j=1}^{r-1} \boldsymbol{a}^{n-p_{j}} f_{j} . \quad$ If $p_{r} \geqslant 1$, since $n-p_{r} \leq n$ - 1, we have $\boldsymbol{a}^{n} \cap \boldsymbol{b}_{r-1} \subseteq \sum_{j=1}^{r-1} \boldsymbol{a}^{n-p_{j}} f_{j}+f_{r}\left(\sum_{j=1}^{r-1} \boldsymbol{a}^{n-p_{r}-p_{j}} f_{j}\right)=\sum_{j=1}^{r-1} \boldsymbol{a}^{n-p_{j}} f_{j}$, and this completes the proof.

COROLlaRY 2.7. If $\boldsymbol{a}$ and $\boldsymbol{b}=\left(f_{1}, \cdots, f_{r}\right)$ are ideals of a local ring $A$, then $f_{1}^{*}, \cdots, f_{r}^{*}$ form $a G_{A}(a)$-sequence if and only if $f_{1}, \cdots, f_{r}$ form an A-sequence and moreover $\boldsymbol{a}^{n} \cap \boldsymbol{b}=\sum_{i=1}^{r} \boldsymbol{a}^{n-p_{i}} f_{i}$ for all $n \geqslant 1$.

Remark 2.8. The following example justifies the hypotheses of the above proposition. Let $A$ be the ring $k[X, Y, Z, T] /\left(X T-Y^{2}, T-Y-T Z\right)$ $=k[x, y, z, t], a=(x, y), f_{1}=x$ and $f_{2}=z$; then it is easy to see that 
$f_{1}, f_{2}$ form a regular sequence and $p_{1}=1, p_{2}=0$. We have : $x t \in \boldsymbol{a}^{2} \cap(x)$, but $x t \notin a x$; on the other hand $\boldsymbol{a} \cap(x, z)=(x)+\boldsymbol{a} \cap(z)=(x)+\boldsymbol{a} z$ and, if $n \geqslant 2$, then $\boldsymbol{a}^{n} \cap(x, z)=\boldsymbol{a}^{n}=\boldsymbol{a}^{n-1} x+\left(y^{n}\right)=\boldsymbol{a}^{n-1} x+\boldsymbol{a}^{n} z$ since $y^{n}=$ $x y^{n-1}+y^{n} z$.

Remark 2.9. The results of the present section extend to a quite general situation the theorem proved by Hironaka $\left(\left[\mathrm{H}_{2}\right]\right.$, Prop. 6) for a local ring $(A, m)$, when $a=m$ and $b=(f)=$ principal ideal, generated by an element in $\boldsymbol{m}-\boldsymbol{m}^{2}$.

\section{Applications}

In this section we discuss some applications of the preceding results.

Proposition 3.1. Let $\boldsymbol{a}$ and $\boldsymbol{b}=\left(f_{1}, \cdots, f_{r}\right)$ be ideals of $A$ such that $\boldsymbol{b} \subseteq \boldsymbol{a}, f_{1}, \cdots, f_{r}$ is an $A$-sequence and $\boldsymbol{a} \boldsymbol{b}=\boldsymbol{a}^{2}$. Then the initial forms of the $f_{i}$ 's form $a G_{A}(a)$-sequence.

Proof. If $f_{i} \in \boldsymbol{a}^{2}=\boldsymbol{a} \boldsymbol{b}$ then we would get a relation of the form

$$
a_{1} f_{1}+\cdots+\left(1+a_{i}\right) f_{i}+\cdots+a_{r} f_{r}=0, \quad a_{j} \in \boldsymbol{a}(\forall j) .
$$

But since $f_{1}, \cdots, f_{r}$ is an $A$-sequence, in any relation $\sum x_{j} f_{j}=0$ all the coefficients $x_{j}$ must lie in the ideal $\left(f_{1}, \cdots, f_{r}\right)$. This is well known and easy to prove by induction on $r$. Thus $f_{i} \notin \boldsymbol{a}^{2}$ and we have $p_{1}=\cdots=$ $p_{r}=1$. Therefore it is enough to prove, by Proposition 2.6, that $\boldsymbol{a}^{n} \cap \boldsymbol{b}$ $=a^{n-1} b$. This is true for $n=1$; if $n \geqslant 2$ we have $a^{n}=a^{n-1} b$, hence $\boldsymbol{a}^{n} \cap \boldsymbol{b}=\boldsymbol{a}^{n-1} \boldsymbol{b} \cap \boldsymbol{b}=\boldsymbol{a}^{n-1} \boldsymbol{b}$.

Remark 3.2. An interesting situation in which we can apply the above proposition is the following: let $(A, m)$ be a local ring of dimension $r$ which is Cohen-Macaulay, with embedding dimension $m$ and multiplicity $e$; then one can show ([S], Theorem 1) that $m \leqslant e+r-1$ and the equality holds if and only if there is an $A$-sequence $f_{1}, \ldots, f_{r}$ in $\boldsymbol{m}$ such that $\boldsymbol{m}^{2}=\boldsymbol{m}\left(f_{1}, \cdots, f_{r}\right)$. The latter equality is exactly our condition on the ideals $\boldsymbol{a}=\boldsymbol{m}$ and $\boldsymbol{b}=\left(f_{1}, \cdots, f_{r}\right)$.

Remark 3.3. The results of the present paper can be used to give a new and simplified proof of ([V], Theorem 3.2), i.e. of the following claim:

Let $A$ be a Cohen-Macaulay ring and let $a_{1}, \cdots, a_{s}$ be a regular 
sequence, $I=\left(a_{1}, \cdots, a_{s}\right), t$ an integer $\geqslant 1$. Then $G_{A}(a)$ is Cohen-Macaulay if $\boldsymbol{a}=\boldsymbol{I}^{t}$.

Proof. As in [V] we may assume that $A$ is a $r$-dimensional local ring with maximal ideal $m$. Let $a_{1}, \cdots, a_{s}, f_{s+1}, \cdots, f_{r}$ be a maximal $A$ sequence in $\boldsymbol{m}$ and let $\boldsymbol{J}=\left(f_{s+1}, \cdots, f_{r}\right), f_{i}=a_{i}^{t}$, for each $i=1, \cdots, s$ and $\boldsymbol{b}=\left(f_{1}, \cdots, f_{r}\right)$. Since $f_{1}, \cdots, f_{s}$ is a regular sequence modulo $J$ we have, by [V], Lemma 2.1, that $\boldsymbol{a}^{n} \cap \boldsymbol{b} \subseteq \boldsymbol{a}^{n-1}\left(f_{1}, \cdots, f_{s}\right)+\boldsymbol{J}$ for all $n \geqslant 1$; furthermore, since $f_{s+1}, \cdots, f_{r}$ is a regular sequence modulo $I$, hence modulo $\boldsymbol{a}^{n}$ for all $n \geqslant 1$, by [R-V], Lemma 1.1, we get: $\boldsymbol{a}^{n} \cap \boldsymbol{J}=\boldsymbol{a}^{n} \boldsymbol{J}$. From this it follows that $\boldsymbol{a}^{n} \cap \boldsymbol{b} \subseteq \boldsymbol{a}^{n} \cap\left(\boldsymbol{a}^{n-1}\left(f_{1}, \cdots, f_{s}\right)+\boldsymbol{J}\right)=\boldsymbol{a}^{n-1}\left(f_{1}\right.$, $\left.\cdots, f_{s}\right)+\boldsymbol{a}^{n} \cap \boldsymbol{J}=\boldsymbol{a}^{n-1}\left(f_{1}, \cdots, f_{s}\right)+\boldsymbol{a}^{n} \boldsymbol{J}$; thus the $f_{i}^{*}$ 's form a $G_{A}(a)-$ sequence by Proposition 2.6. Since $\operatorname{dim} G_{A}(a)=r$, by $[M-R]$ this is enough to prove that $G_{A}(a)$ is Cohen-Macaulay.

We conclude the paper trying to compare the initial forms of a set of elements with respect to two different ideals $\boldsymbol{a}$ and $\boldsymbol{I}$.,

First of all, it is easy to see that the initial form of the same element with respect to two different ideals may or may not be a 0 divisor; for instance, if $A=k[[x, y, z]]=k[[X, Y, Z]] /\left(X Y-Z^{2}\right), \quad a=$ $(x, y, z), I=(x, z)$, then it is clear that the initial form of $x$ relative to $\boldsymbol{a}$ is a non 0-divisor, while the initial form with respect to $I$ is a 0 divisor. Therefore $(x)^{*}=\left(x^{*}\right)$ in $G_{A}(a)$ (Proposition 2.1); on the contrary $(x)^{*}$ in $G_{A}(I)$ is not generated by the initial form of $x$, because of Theorem 1.1 .

In the following proposition we denote by $f^{*}$ the initial form with respect to $\boldsymbol{a}$ and by $f^{0}$ the initial form with respect to $I$.

Proposition 3.4. Let $I \subseteq \boldsymbol{a}$ be ideals of $A$ and let $f_{1}, \cdots, f_{r}$ be elements of $I$ such that $v_{I}\left(f_{i}\right)=v_{a}\left(f_{i}\right)$ for each $i$. Assume that $f_{1}^{*}, \cdots$, $f_{r}^{*}$ form a $G_{A}(a)$-sequence. Then $f_{1}^{0}, \cdots, f_{r}^{0}$ form a minimal base of the ideal $\left(f_{1}^{0}, \cdots, f_{r}^{0}\right)$ of $G_{A}(I)$.

Proof. By [A-B], Corollary 2.9, $f_{1}^{*}, \cdots, f_{r}^{*}$ is a $G_{A}(a)$-sequence in any order. Now if $f_{r}^{0}=\sum_{i=1}^{r-1} a_{i}^{0} f_{i}^{0}$, let $a=\sum_{i=1}^{r-1} a_{i} f_{i}$ and $p=v_{a}\left(f_{r}\right)=$ $v_{I}\left(f_{r}\right)$; then $f_{r}=a+b$, where $b \in \boldsymbol{I}^{p+1}$. Hence $a \in \boldsymbol{a}^{p}$ and $a \notin \boldsymbol{a}^{p+1}$; it follows that $f_{r}^{*}=a^{*} \in\left(f_{1}, \cdots, f_{r-1}\right)^{*}=\left(f_{1}^{*}, \cdots, f_{r-1}^{*}\right)$, which is absurd. 


\section{REFERENCES}

[A-B] M. Auslander and D. Buchsbaum, Codimension and multiplicity, Ann. of Math., 68 (1958), 625-657.

$\left[\mathrm{H}_{1}\right] \quad \mathrm{H}$. Hironaka, Resolution of singularities of an algebraic variety over a field of characteristic, 0. Ann. of Math., 79 (1964), 109-326.

$\left[\mathrm{H}_{2}\right]$ H. Hironaka, Certain numerical characters of singularities, J. Math. Kyoto Univ., 10 (1970), 151-187.

[M-R] J. Matijevich and P. Roberts, A conjecture of Nagata on graded CohenMacaulay rings, J. Math. Kyoto Univ., 14 (1974), 125-128.

[R] L. J. Ratiff, Jr., On quasi unmixed local domains, the altitude formula, and the chain condition for prime ideals (II), Amer. J. Math., 92 (1970), 99-144.

[R-V] L. Robbiano and G. Valla, On normal flatness and normal torsion freeness, J. of Algebra, 43 (1976), 552-560.

[S] J. Sally, On the associated graded ring of a local Cohen-Macaulay ring, J. Math. Kyoto Univ., 17 (1977), 19-21.

[V] G. Valla, Certain graded algebras are always Cohen-Macaulay, J. of Algebra, 42 (1976), 537-548.

Istituto matematico-Politecnico di Torino

Istituto matematico-Università di Genova 